\title{
BMJ Open Accelerated induction regimens of TNF- alpha inhibitors in patients with inflammatory bowel disease: a scoping review protocol
}

\author{
Amy Johnston, ${ }^{1}$ Sabrina Natarajan, ${ }^{2}$ Meghan Hayes, ${ }^{2}$ Erika MacDonald, ${ }^{2}$ \\ Risa Shorr ${ }^{3}$
} Natarajan S, Hayes M, et al. of TNF-alpha inhibitors in patients with inflammatory bowel disease: a scoping review protocol. BMJ Open 2018;8:e019909. doi:10.1136/ bmjopen-2017-019909

\section{- Prepublication history and} additional material for this paper are available online. To view these files, please visit the journal online (http://dx.doi. org/10.1136/bmjopen-2017019909).

Received 3 October 2017 Revised 7 December 2017 Accepted 19 December 2017
To cite: Johnston A, Accelerated induction regimens

\section{ABSTRACT}

Introduction Tumour necrosis factor (TNF)-alpha inhibitors are commonly used to treat inflammatory bowel disease (IBD). In patients with IBD who are unresponsive to their first induction dose, the implementation of an 'accelerated' induction dose schedule (doses more frequent than recommended in product monographs) is becoming increasingly common. It is unclear whether this practice results in favourable patient outcomes, such as avoidance of surgery and disease remission. As such, there is a need to identify and map the current evidence base on accelerated induction schedules of these medications in the treatment of IBD.

Methods and analysis A scoping review will be employed to systematically identify and characterise the nature of scientific literature on accelerated induction regimens of TNF-alpha inhibitors. MEDLINE, Embase, International Pharmaceutical Abstracts and grey literature will be searched to identify relevant studies. The titles/ abstracts of all records and full text of potentially relevant articles will be independently screened for inclusion by two reviewers. Data will be abstracted from included studies by one reviewer and verified for accuracy by another. The findings will be synthesised descriptively.

Ethics and dissemination We intend to report the findings of this scoping review in a peer-reviewed journal and a scientific conference.

Trial registration This research was registered prospectively with the Open Science Framework (https:// osf.io/z7n2d/).

\section{BACKGROUND}

\section{Introduction}

${ }^{1}$ Cardiovascular Research Methods Centre, University of Ottawa Heart Institute, Ottawa, Ontario, Canada

${ }^{2}$ Pharmacy Department, The Ottawa Hospital, Ottawa, Ontario, Canada

${ }^{3}$ Learning Services, The Ottawa Hospital, Ottawa, Ontario, Canada

Correspondence to Amy Johnston; ajohnston@ottawaheart.ca
Inflammatory bowel disease (IBD) is an umbrella term used to describe a complex set of chronic relapsing and remitting inflammatory conditions characterised by severe inflammation of the gastrointestinal mucosa. ${ }^{1-3}$ The two major categories of IBD are Crohn's disease (CD) and ulcerative colitis (UC), which are distinguished from each other by clinical symptoms and location. ${ }^{1}$ Whereas $\mathrm{CD}$ can affect any part of the gastrointestinal tract, the gastrointestinal
Strengths and limitations of this study

We will conduct a comprehensive literature search of peer-reviewed and grey literature across several databases enabling us to identify both unpublished and difficult to locate studies.

- Our scoping review will be undertaken using a systematically rigorous approach, guided by methodology outlined by Arksey and 0'Malley and the Joanna Briggs Institute.

No restrictions will be placed on language.

- To increase the feasibility of our review, data will be abstracted by one reviewer and independently verified by another reviewer.

- While similar study designs and types of documents will be compared against each other, the quality of evidence will not be assessed in keeping with conventional scoping review methodology.

inflammatory effects of UC are limited to the large intestine. ${ }^{4}$

As IBD is a chronic inflammatory disease for which there is no cure, therapies aim to induce and maintain control of symptoms, minimise complications and improve quality of life. ${ }^{156}$ In addition to lifestyle changes, current practice guidelines recommend the use of a variety of drug therapies in the treatment of IBD, including: 5-aminosalicylic acid agents, immune-modulating agents such as azathioprine or methotrexate, corticosteroids and biologic agents (monoclonal antibodies), including those designed to inhibit the cell signalling protein tumour necrosis factor (TNF)-alpha. ${ }^{1-9}$ Indeed, the advent of TNF-alpha inhibitors brought about a substantial shift in IBD therapy over the past 20 years. Today, these drugs are commonly used when patients fail to maintain remission of symptoms with other agents or require high doses of corticosteroids to control symptoms. ${ }^{15}$ TNF-alpha inhibitors can allow patients to avoid or delay the need for surgical 
interventions including the formation of an ostomy, resection of the gastrointestinal tract or colectomy. ${ }^{10}$

Infliximab was the first TNF-alpha inhibitor approved for the treatment of CD. ${ }^{11}$ In 2002, Hanauer et alpublished A Crohn's Disease Clinical Trial Evaluating Infliximab in a New Long-Term Treatment Regimen (in patients with moderately to severely active Crohn's disease) (ACCENT I) study in which the authors evaluated the efficacy of repeated doses of infliximab to maintain remission in patients with moderate-to-severe non-fistulising CD. ${ }^{12}$ Patients who received infliximab were found to have longer maintenance of remission compared with those who received placebo. Subsequent studies illustrated the efficacy of infliximab in fistulising CD (A Crohn's Disease Clinical Trial Evaluating Infliximab in a New Long-Term Treatment Regimen (in patients with fistulizing Crohn's disease) (ACCENT II) trial) ${ }^{13}$ and in maintaining remission in UC (Active Ulcerative Colitis Trials 1 and 2). ${ }^{14}$ Although the indication for TNF-alpha inhibitors is moderate-to-severe IBD, most patients included in these studies were diagnosed with moderate disease. ${ }^{12} 14$ Notably, patients with severe UC requiring ongoing highdose corticosteroids were specifically excluded from the ACT 1 and 2 trials. ${ }^{14}$ Since the publication of these landmark studies, infliximab has become a key agent in the treatment of IBD; however, other TNF-alpha inhibitors such as adalimumab, golimumab and certolizumab have subsequently been approved for this indication. More recently, TNF-alpha inhibitor biosimilars (medications designed to have the same active properties as, and no clinically meaningful differences when compared with existing TNF-alpha inhibitor "reference products' ${ }^{15}$ ) as well as interleukin and integrin inhibitors have also been approved for the treatment of IBD. ${ }^{16-18}$

Dosing of TNF-alpha inhibitors requires an induction phase and a maintenance phase. In the induction phase, two or three doses of the TNF-alpha inhibitor are given within a few weeks to improve clinical symptoms. ${ }^{19}$ In the maintenance phase, the TNF-alpha inhibitor is administered at regular intervals to maintain control of symptoms and adjunctive medications are often continued. The dose can be increased to treat worsening symptoms. ${ }^{20-24}$ Induction doses of TNF-alpha inhibitors can also be escalated in patients with poor or incomplete response to the initial induction doses. ${ }^{13}$

In 2015, Gibson et al published a study examining whether patients with acute severe UC required more frequent or higher infliximab doses to overcome the higher levels of inflammation and faster drug clearance noted in this population. ${ }^{25}$ In their retrospective study of 50 hospitalised patients with acute severe UC, 15 received what the authors termed an 'accelerated infliximab induction regimen': three doses of infliximab within a median of 24 days rather than the usual 6 weeks. Although this was a small study, in the 12-month period after induction there was a statistically significant difference in the number of colectomies between the group who received the accelerated regimen compared with those who received the standard induction regimen $(6.7 \%$ vs $40 \%$, $\mathrm{P}=0.039$ ). This difference, however, was not maintained after long term follow-up (2 years).

\section{Rationale}

It is unclear whether accelerated TNF-alpha inhibitor induction dosing regimens result in favourable patient outcomes (eg, decreased rates of surgical intervention and increased rates of disease remission) as studies examining the practice's safety and efficacy do not appear to be well documented in the primary literature. Safety data, including the degree of immunosuppression, potential risk of malignancy, hepatotoxicity and antibody formation also appear to be scarce. It is, therefore, difficult to weigh the potential benefits and risks of implementing these dosing regimens in patients with IBD.

A 2008 health technology inquiry by the Canadian Agency for Drugs and Technologies in Health revealed no relevant clinical studies, health technology assessments or literature reviews on this topic. ${ }^{26}$ Since that time, accelerated dosing schedules have become increasingly implemented in inpatient settings. ${ }^{27}{ }^{28}$ Given the increasing global incidence and prevalence of IBD as well as the high cost of TNF-alpha inhibitors, the practice of accelerated dose scheduling, which involves prescribing medication more quickly than is currently recommended in product monographs, will put an even greater strain on hospital resources. ${ }^{29}$ It is, therefore, prudent to systematically identify and map the extent, range and nature of current research on this practice in the treatment of IBD.

\section{METHODOLOGY}

\section{Study design}

Scoping review methodology will be used to systematically identify and map the nature of evidence on this topic. As a guide, we will use the methodological framework described by Levac et $a l^{30}$ and the Joanna Briggs Institute (JBI) Reviewers' Manual on Methodology for JBI Scoping Reviews, ${ }^{31}$ both of which build on the methods developed by Arksey and O'Malley. ${ }^{32}$

This review will be completed in six stages by three pharmacists (SN, MH, EM), a medical librarian (RS) and a researcher (AJ). As part of stage one, the initial research questions have been developed. Stage two will involve the identification of relevant studies. As a third stage, we will select studies for inclusion and, in the fourth stage, we will extract relevant data from included studies. In the fifth stage of this review, we will synthesise and report our findings. Throughout the review process, we will engage in stakeholder consultation (stage six) with clinical specialists to gain insight on aspects about the research topic that the literature does not reveal.

\section{Protocol}

This protocol was developed using The Preferred Reporting Items for Systematic Reviews and Meta-Analyses for Protocols (PRISMA-P, online supplementary 
Box Population, concept and context elements of the study eligibility criteria on this topic

Population
Patients of all ages and any sex diagnosed with inflammatory bowel
disease.
Concept
Treatment with any tumour necrosis factor (TNF)-alpha inhibitor
using any 'accelerated' induction phase dosing schedule.
All reported comparisons of interest will be considered.
All primary and secondary outcomes as reported by study authors
are of interest; we will not screen records for inclusion based on
outcomes reported.
Context
We will include experimental and observational study designs
that attempt to evaluate the efficacy and/or safety of using a TNF-
alpha inhibitor induction regimen with induction phase doses given
more frequently than what is specified in the most current product
monograph (as determined by the region in which the study was
conducted, eg, Canada, USA or the European Union).
Evidence reported in research syntheses (systematic reviews, meta-
analyses and narrative reviews) will also be considered.
Conference abstracts will be included if full-text publications are
not available.
No date or language restrictions will be placed on the literature
search.

appendix 1) ${ }^{33}$ It was reviewed and revised by all members of the research team as required. The research was registered prospectively with the Open Science Framework on 31 August 2017 (https://osf.io/z7n2d/).

\section{Research questions}

The objective of this review is to summarise the nature and extent of available evidence and research activity relating to accelerated induction regimens of TNF-alpha inhibitors in the treatment of IBD. For the purposes of this review, 'accelerated' is defined as any TNF-alpha inhibitor induction period dosing frequency that exceeds what is recommended in the most current product monograph. To fulfil this objective, this research will be guided by the following primary research question, 'What studies have evaluated, and/ or have been published on, accelerated induction regimens of TNF-alpha inhibitors in the treatment of IBD?' Secondary research questions include:

- What kinds of accelerated induction dose schedules have been studied in the literature?

- What clinical indications (eg, IBD severity) have warranted the use of the accelerated regimens in the literature?

- What primary and secondary outcomes have been used to evaluate the efficacy and safety of accelerated regimens in the literature?

All research questions were developed and refined in collaboration with the entire research team.

\section{Literature search}

Scoping reviews aim to search broadly and extensively for available literature on a topic; however, certain guiding parameters are required to help direct and increase the feasibility of the overall search. The draft MEDLINE literature search strategy (online supplementary appendix 2) was designed by a medical librarian (RS), in collaboration with the research team, to be broad yet sensitive in capturing relevant literature by combining relevant keywords related to the study population, concept and context of interest (box). Specifically, the strategy combined elements from three concepts:

1. Participants with IBD (eg, ibd or inflammat* bowel or colitis or crohn*),

2. TNF-alpha inhibitor medications, broadly (eg, tnf alpha adj (inhibit* or block* or antagonist*) and specifically (eg, Remicade or Remsima or Inflectra or infliximab),

3. Dosing scheme (eg, dose and dosage in combination with related MeSH subject headings) to appear in author-supplied keywords, titles and abstracts.

The search strategy will be tested and refined using an iterative process to facilitate a robust and comprehensive search across other key databases (eg, Cochrane CENTRAL via Wiley and International Pharmaceutical Abstracts). No date or language restrictions will be applied to searches.

A search for grey literature will also be conducted and will include: conference abstracts, clinical trial registries as well as targeted internet searches. We will also consult clinical experts to identify known studies that address this research topic as well as any key conferences and/or journals that may not be indexed. The reference lists of included articles will be hand searched to identify additional studies of interest. Mendeley ${ }^{34}$ reference management and pdf organiser software will be used to manage records identified from all searches.

To validate the initial search strategy, search results will be cross-referenced against a list of relevant studies known to the research team. The validated search, as well as any subsequent search completed in additional databases, may be modified and repeated in the early stages of the review if new information emerges to facilitate the completion of a comprehensive literature search. Any modifications to a search strategy will be documented on our Open Science Framework registry and justification will be provided in the final publication of findings.

\section{Study selection}

While the aim of scoping reviews is to obtain a broad, iterative, examination of a topic of interest, some constraints on inclusion are required to ease the process of identifying the most appropriate literature.$^{32}$ As such, relevant studies will be selected for inclusion by screening all identified records against a set of a priori eligibility criteria as defined in box.

As suggested by Levac et $a l^{30}$ we will employ a team approach to the selection of included studies. First, two 
Table 1 Key information to be charted during the review process

\begin{tabular}{|c|c|c|c|}
\hline Study characteristics & Participant characteristics & $\begin{array}{l}\text { Intervention and } \\
\text { comparator(s) }\end{array}$ & Key findings \\
\hline $\begin{array}{l}\text { Authors } \\
\text { Trial name } \\
\text { Publication year } \\
\text { Country of origin } \\
\text { Primary and secondary } \\
\text { objectives } \\
\text { Study design } \\
\text { Type of intervention } \\
\text { Study sponsor/funding agency }\end{array}$ & $\begin{array}{l}\text { Inclusion and exclusion criteria } \\
\text { Total number of participants } \\
\text { Total number of females/males } \\
\text { Mean age } \\
\text { IBD diagnosis and diagnostic criterion } \\
\text { Disease severity (eg, mean CDAl score) } \\
\text { and duration } \\
\text { Previous surgery } \\
\text { Concomitant medication }\end{array}$ & $\begin{array}{l}\text { Duration of } \\
\text { intervention (if } \\
\text { applicable) } \\
\text { Type of accelerated } \\
\text { induction phase } \\
\text { dosing schedule } \\
\text { Comparator(s) }\end{array}$ & $\begin{array}{l}\text { Results of primary } \\
\text { outcome } \\
\text { Results of } \\
\text { secondary } \\
\text { outcomes }\end{array}$ \\
\hline
\end{tabular}

CDAI, Crohn's Disease Activity Index; IBD, inflammatory bowel disease.

reviewers will independently complete a pilot screening exercise on a random sample of titles and abstracts of all types of records retrieved. Once completed, the review team will meet to discuss challenges and questions that arose during the pilot test and, if necessary, adjustments to eligibility criteria will be made in consultation with the research team. When all reviewers are satisfied with the results of the pilot test, screening of titles and abstracts of all records retrieved by the database and grey literature searches will then be completed independently by two reviewers. The research team will meet at the midpoint and final stages of this process to discuss any questions that arise throughout the screening process.

Any full-text records deemed potentially eligible for inclusion after the title/abstract screening stage will be retrieved and reviewed for eligibility by two independent reviewers. Discrepancies in inclusion will be resolved by discussion and consensus. Decisions that cannot be made through discussion will be brought to a third reviewer for review. Covidence (version 2018), a web-based systematic review software program (http://www.covidence.org), will be used to facilitate the study selection process.

Abstracts or included full-text articles written in English and French will be screened and/or extracted by members of the research team. We will use our extensive personal network of native or fluent foreign language speakers to assist in the management of articles written in other languages. If required, and if possible, we will use Google Translate (https://translate.google.ca/) to assist in translating articles written in languages outside those available in our personal network. Although there are inherent limitations in using this resource, it will facilitate a broad inclusion of literature that might otherwise be overlooked. ${ }^{35}$

\section{Data extraction}

The entire research team will be involved in the development, and refinement, of the final data extraction form; however, we have conducted a preliminary exercise to develop a small number of a priori data items, across four distinct categories, to be charted (table 1). These items have been incorporated into a standard extraction form.
Two reviewers will independently pilot the form on a random sample of 5-10 included studies. Once completed, the research team will meet to review and revise the extraction form as necessary. As the extraction process continues, additional data of relevance may be identified that was not defined a priori. Given the iterative nature of the extraction process, we expect the form to be continually updated as the review progresses. ${ }^{30} 31$ To assist with the feasibility of this review, Covidence systematic review software will be used to extract data from included studies and will be completed independently by one reviewer and verified for accuracy by another.

\section{Quality assessment}

Our aim is to map and describe the current available evidence on this topic, not to collect and analyse the best available evidence for the purposes of addressing a specific research question. As such, we will not formally appraise the methodological quality of included studies, or grade the evidence, as these types of activities are infrequently undertaken as part of the scoping review process. ${ }^{31} \mathrm{We}$ will, however, provide an overview of the level of evidence available on this topic based on included study design.

\section{Data synthesis and reporting of findings}

Details of the literature search and screening results will be summarised narratively and will be presented using a PRISMA flow diagram. ${ }^{36}$ We will also provide a comprehensive descriptive table of studies that fully addressed the research questions. Specific data items (ie, study and participant characteristics) will also be collated and summarised in tables. To present a visual and descriptive summary of key results and descriptive data, we will use diagrams, such as a word cloud (Wordle.com) and a pictorial summary of the types of accelerated doses identified. Descriptive data will be synthesised using qualitative thematic analysis techniques by one reviewer and verified by another.

\section{Stakeholder consultation}

We will engage in ongoing stakeholder consultation throughout the progression of this review. Given the potential implication of this research on clinical practice, 
we feel it pertinent to engage with clinical experts beginning early in the review process as a part of the knowledge translation process.

\section{CONCLUSIONS}

This scoping exercise will allow for a broad, iterative examination of the current state of evidence on accelerated induction regimens of TNF-alpha inhibitors in the treatment of IBD. We will employ the same systematic and rigorous methodology to retrieve and extract data as that used in the undertaking of a traditional systematic review and aim to identify knowledge gaps in this area to determine whether a more in-depth knowledge synthesis is warranted.

Contributors All authors made substantial contributions to the conception and design of the work, drafting the work and revising it critically for important intellectual content and providing final approval of the version to be published and agree to be accountable for all aspects of the work in ensuring that questions related to the accuracy or integrity of any part of the work are appropriately investigated and resolved.

Funding This research received no specific grant from any funding agency in the public, commercial or not-for-profit sectors.

Competing interests None declared.

Patient consent Obtained.

Provenance and peer review Not commissioned; externally peer reviewed.

Open Access This is an Open Access article distributed in accordance with the Creative Commons Attribution Non Commercial (CC BY-NC 4.0) license, which permits others to distribute, remix, adapt, build upon this work non-commercially, and license their derivative works on different terms, provided the original work is properly cited and the use is non-commercial. See: http://creativecommons.org/ licenses/by-nc/4.0/

(C) Article author(s) (or their employer(s) unless otherwise stated in the text of the article) 2018. All rights reserved. No commercial use is permitted unless otherwise expressly granted.

\section{REFERENCES}

1. Lahad A, Weiss B. Current therapy of pediatric Crohn's disease. World J Gastrointest Pathophysiol 2015;6:33-42.

2. de Vries HS, van Oijen MG, Driessen RJ, et al. Appropriate infliximab infusion dosage and monitoring: results of a panel meeting of rheumatologists, dermatologists and gastroenterologists. Br J Clin Pharmacol 2011;71:7-19.

3. Karp LC, Targan SR. Heterogeneity of inflammatory bowel diseases. In: Targan SR, Shanahan F, Karp LC, eds. Inflammatory bowel disease: translating basic science into clinical practice. West Sussex, UK: Blackwell Publishing Ltd, 2010.

4. Kasper DL, Fauci AS, Hauser SL, et al. Chapter 148: inflammatory bowel diseases. Harrison's manual of internal medicine. Toronto, Ontario, Canada: McGraw-Hill Education, 2016:790-4.

5. Abraham C, Cho JH. Inflammatory bowel disease. N Engl J Med 2009;361:2066-78.

6. Sandborn W, Schreiber S, Hanauer S, et al. Long-term maintenance of remission with no dose escalation after re-induction with certolizumab pegol in patients with crohn's disease exacerbation who were naive to anti-tnf treatment: 4-year results from the precise 4 study. Am J Gastroenterol 2010;105:S418.

7. Terdiman JP, Gruss CB, Heidelbaugh JJ, et al. American Gastroenterological Association Institute Guideline on the use of thiopurines, methotrexate, and anti-TNF- $\alpha$ biologic drugs for the induction and maintenance of remission in inflammatory Crohn's disease. Gastroenterology 2013;145:1459-63.

8. Kornbluth A, Sachar DB. Practice Parameters Committee of the American College of Gastroenterology. Ulcerative colitis practice guidelines in adults: American College of Gastroenterology, Practice Parameters Committee [correction appears in: Am J Gastroenterol 2010; 105: 500]. Am J Gastroenterol 2010;105:501-23.
9. Biancone L, Annese V, Ardizzone S, et al. Safety of treatments for inflammatory bowel disease: Clinical practice guidelines of the Italian Group for the Study of Inflammatory Bowel Disease (IG-IBD). Dig Liver Dis 2017;49:338-58.

10. Surlin V, Copaescu C, Saftoiu A. An update to surgical management of inflammatory bowel diseases. In: Szabo I, ed. Inflammatory bowel disease. Croatia: In Tech, 2012.

11. Loftus EV. New data on the use of biologic agents for Crohn's disease and ulcerative colitis: highlights from the 2009 CCFA advances in IBD meeting. Gastroenterol Hepatol 2010;6:4-16.

12. Hanauer SB, Feagan BG, Lichtenstein GR, et al. Maintenance infliximab for Crohns disease: the ACCENT I randomised trial. Lancet 2002;359:1541-9.

13. Sands BE, Anderson FH, Bernstein CN, et al. Infliximab maintenance therapy for fistulizing Crohn's disease. N Engl J Med 2004;350:876-85.

14. Rutgeerts P, Sandborn WJ, Feagan BG, et al. Infliximab for induction and maintenance therapy for ulcerative colitis. $N$ Engl J Med 2005;353:2462-76

15. U.S. Food and Drug Administration. Information on biosimilars. 2016. https://www.fda.gov/drugs/developmentapprovalprocess/ howdrugsaredevelopedandapproved/approvalapplications/ therapeuticbiologicapplications/biosimilars/.

16. Côté-Daigneault J, Bouin M, Lahaie R, et al. Biologics in inflammatory bowel disease: what are the data? United European Gastroenterol J 2015;3:419-28.

17. Hlavaty T, Letkovsky J. Biosimilars in the therapy of inflammatory bowel diseases. Eur J Gastroenterol Hepatol 2014;26:1-7.

18. Wu S, Yang YM, Zhu J, et al. Meta-analysis of efficacy and safety of new oral anticoagulants compared with uninterrupted vitamin $\mathrm{K}$ antagonists in patients undergoing catheter ablation for atrial fibrillation. Am J Cardiol 2016;117:926-34.

19. Schwartz M, Cohen R. Optimizing conventional therapy for inflammatory bowel disease. Curr Gastroenterol Rep 2008;10:585-90.

20. Humira [Product Monograph]. Saint-Laurent, QC: AbbVie Corporation, 2014.

21. Simponi [Product Monograph]. Toronto, ON: Janssen Inc, 2017.

22. Remicade [Product Monograph]. Toronto, ON: Janssen Inc, 2017.

23. European Medicines Agency. Humira. 2017. http://www.ema.europa. eu/docs/en_GB/document_library/EPAR_-_Product_Information/ human/000481/WC500050870.pdf.

24. European Medicines Agency. Remicade. 2016. http://www.ema. europa.eu/docs/en_GB/document_library/EPAR_-_Product_ Information/human/000240/WC500050888.pdf.

25. Gibson DJ, Heetun ZS, Redmond CE, et al. An accelerated infliximab induction regimen reduces the need for early colectomy in patients with acute severe ulcerative colitis. Clin Gastroenterol Hepatol 2015;13:330-5

26. Compressed dosing schedule for adalimumab and infliximab for the treatment of inflammatory bowel diseases : clinical and costeffectiveness. Ottawa, Canada: Health Technology Inquiry Service, 2008.

27. University of North Carolina. UNC inflammatory bowel disease drug protocol infliximab (Remicade). Chapel Hill: University of North Carolina School of Medicine, 2016. (cited 20 Sep 2017).

28. Rofaiel R, Kohli S, Mura M, et al. A 53-year-old man with dyspnoea, respiratory failure, consistent with infliximab-induced acute interstitial pneumonitis after an accelerated induction dosing schedule. BMJ Case Rep 2017. doi: 10.1136/bcr-2017-219956. [Epub ahead of print 12 May 2017].

29. Ye Y, Pang Z, Chen $W$, et al. The epidemiology and risk factors of inflammatory bowel disease. Int J Clin Exp Med 2015;8:22529-42.

30. Levac D, Colquhoun H, OBrien KK. Scoping studies: advancing the methodology. Implement Sci 2010;5:69.

31. The Joanna Briggs Institute. Joanna briggs institute reviewers' manual 2015: methodology for JBI scoping reviews. South Australia : The Joanna Briggs Institute, The University of Adelaide, 2015.

32. Arksey H, OMalley L. Scoping studies: towards a methodological framework. Int J Soc Res Methodol 2005;8:19-32.

33. Moher D, Shamseer L, Clarke M, et al. Preferred reporting items for systematic review and meta-analysis protocols (PRISMA-P) 2015 statement. Syst Rev 2015;4:1.

34. Mendeley reference management software \& researcher network. Amsterdam, The Netherlands: Elsevier. http://www.mendeley.com

35. Balk EM, Chung M, Chen ML, et al. Assessing the accuracy of google translate to allow data extraction from trials published in non-english languages [Internet]. Rockville, MD: Agency for Healthcare Research and Quality (US), 2013.

36. Moher D, Liberati A, Tetzlaff $J$, et al. Preferred reporting items for systematic reviews and meta-analyses: the PRISMA statement. Ann Intern Med 2009;151:264-9. 